\title{
tic\&société
}

Vol. 7, N 2 | 2ème semestre 2013

Mondes numériques : nouvelles perspectives de la recherche

\section{Par-delà la dichotomie public/privé : la mise en visibilité des pratiques numériques et ses enjeux éthiques}

\section{Guillaume LATZKO-TOTH and Madeleine PASTINELLI}

\section{OpenEdition}

\section{Journals}

Electronic version

URL: http://journals.openedition.org/ticetsociete/1591

DOI: 10.4000/ticetsociete.1591

\section{Publisher}

Association ARTIC

\section{Electronic reference}

Guillaume LATZKO-TOTH and Madeleine PASTINELLI, « Par-delà la dichotomie public/privé : la mise en visibilité des pratiques numériques et ses enjeux éthiques », tic\&société [Online], Vol. 7, N 2 | 2ème semestre 2013, Online since 01 June 2014, connection on 19 April 2019. URL : http:// journals.openedition.org/ticetsociete/1591 ; DOI : 10.4000/ticetsociete.1591 


\title{
Par-delà la dichotomie public/privé : la mise en visibilité des pratiques numériques et ses enjeux éthiques
}

\author{
Guillaume LATZKO-TOTH \\ Département d'information et de communication \\ Faculté des lettres et des sciences humaines \\ Université Laval \\ Guillaume.Latzko-toth@com.ulaval.ca \\ Madeleine PASTINELLI \\ Département de sociologie \\ Faculté des sciences sociales \\ Université Laval \\ Madeleine.Pastinelli@soc.ulaval.ca
}

Guillaume LATZKO-TOTH est professeur au sein du Département d'information et de communication de l'Université Laval et codirecteur du Laboratoire de communication médiatisée par ordinateur (LabCMO). Ses recherches et publications récentes portent sur l'histoire de l'informatique «sociale» (social computing) dans une perspective sociotechnique, sur les usages des médias numériques émergents, et sur les aspects méthodologiques et éthiques de la recherche en ligne. II est membre du Centre interuniversitaire de recherche sur la science et la technologie (CIRST) et cofondateur de la section « Technologie et médias émergents » de l'Association canadienne de communication (ACC).

Madeleine PASTINELLI est ethnologue de formation, professeure au Département de sociologie de l'Université Laval et membre régulier du CELAT. Chercheure de terrain, elle s'est intéressée à l'identité individuelle, au rapport à l'autre et au développement de liens affinitaires dans différents types d'espace de sociabilité en ligne. Depuis quelques années, elle s'intéresse plus particulièrement aux espaces électroniques qui regroupent des personnes autour de pratiques ou d'identités marginales ou déviantes et s'intéresse aux enjeux normatifs de la multiplication de ce genre de communautés. En parallèle à ces travaux, elle poursuit depuis plusieurs années la réflexion sur les enjeux méthodologiques de l'enquête de terrain en ligne. En collaboration avec deux autres collègues, elle poursuit actuellement une recherche sur l'usage de Facebook, la circulation et la réception de l'information dans les médias sociaux, laquelle recherche a pris la forme d'une étude de cas autour des grèves étudiantes qu'a connues le Québec en 2012. 


\section{Par-delà la dichotomie public/privé : la mise en visibilité des pratiques numériques et ses enjeux éthiques}

Résumé : La question du caractère privé ou public des données occupe une place centrale dans les débats et discussions autour des enjeux éthiques de la recherche sur les pratiques numériques. Dans le contexte d'un encadrement de plus en plus contraignant des recherches en sciences humaines et sociales au chapitre de l'éthique, mais aussi pour outiller les chercheurs désireux d'y voir plus clair, il nous est apparu nécessaire de nous interroger sur les fondements des discours normatifs en éthique de la recherche en ligne s'appuyant sur la dichotomie public/privé. Nous avançons que celle-ci est peu appropriée pour penser les pratiques numériques et les enjeux relatifs au bien-être des sujets sociaux impliqués dans la recherche. Nous proposons plutôt de considérer le degré de publicité des données recueillies, et de nous préoccuper des torts que l'amplification de cette publicité peut engendrer, qu'elle soit le fait des chercheurs, des acteurs impliqués, ou de tiers. Ce faisant, cet article entreprend de proposer un cadre conceptuel commun pour penser les pratiques des acteurs et des chercheurs.

Mots-clés : pratiques numériques, dichotomie public/privé, degré de publicité, éthique de la recherche, visibilité.

Abstract: The private or public status of data is in the midst of debates and discussions about ethical issues raised by research on digital practices. In the context of an increasingly stringent regulation of research in social sciences and humanities, but also in order to provide tools to researchers looking for clearer guidelines in online research ethics, it seemed necessary to question the grounds of normative discourses that are based on the public/private dichotomy. We argue that it is hardly suited to conceptualize digital practices and issues related to the well-being of social subjects involved in the research. Instead, we propose to consider the degree of publicity of collected data, and to be concerned with the harm that an amplification of that publicity can generate, be it caused by researchers, involved actors, or third parties. In so doing, this paper takes on the task of proposing a common conceptual framework to think practices of both actors and researchers.

Keywords: digital practices, public/private dichotomy, degree of publicity, research ethics, visibility. 
Par-delà la dichotomie public/privé :

la mise en visibilité des pratiques numériques et ses enjeux éthiques

Resumen: La cuestión del carácter privado o público de los datos ocupa un lugar central en los debates y discusiones en torno a los desafíos éticos de la investigación sobre las prácticas digitales. En el contexto de marcos reguladores cada vez más restrictivos para las investigaciones en ciencias humanas y sociales en términos éticos, pero también con el propósito de brindar herramientas a los investigadores que desean tener una mayor claridad al respecto, nos parece necesario interrogarnos sobre los fundamentos de los discursos normativos de la ética de la investigación en línea sustentados sobre la dicotomía público/privado. Argumentamos que esta última es poco apropiada para pensar las prácticas digitales y las cuestiones relativas al bienestar de los sujetos sociales involucrados en la investigación. En cambio, proponemos considerar el grado de publicidad de los datos recolectados, y preocuparnos por los perjuicios que la amplificación de esta publicidad -ya sea provocada por los investigadores, los actores implicados o por terceros- puede producir. De este modo, el artículo busca proponer un marco conceptual común para pensar las prácticas de los actores y los investigadores.

Palabras claves: prácticas digitales, dicotomía público/privado, grado de publicidad, ética de la investigación, visibilidad. 


\section{Guillaume LATZKO-TOTH et Madeleine PASTINELLI}

\section{Introduction $^{1}$}

La frontière naguère intuitive entre ce qui relève de la vie privée et de l'expression dans la sphère publique est mise à mal par les nouvelles formes d'interaction médiatisée par ordinateur. Or, la question du caractère privé ou public des objets d'analyse occupe une place centrale dans les débats et discussions entourant les enjeux éthiques de la recherche en ligne. Si cette question s'avère si importante, c'est notamment parce qu'elle est étroitement liée à la question des limites à l'intérieur desquelles la recherche est éthiquement acceptable, si le chercheur n'est pas en mesure d'obtenir le consentement à participer à la recherche de ceux qui en sont l'objet. Dans le contexte d'un encadrement de plus en plus contraignant des recherches en sciences humaines et sociales au chapitre de l'éthique (Trudel et Jean, 2010 ; Laurens et Neyrat, 2010), mais aussi pour outiller les chercheurs désireux d'y voir plus clair, il nous est apparu nécessaire, dans un premier temps, de nous interroger sur les fondements des discours normatifs en éthique de la recherche en ligne s'appuyant sur la distinction public/privé.

L'enjeu est en effet de taille à l'heure où la plupart des pays occidentaux ont adopté ou sont en passe de se doter de politiques d'encadrement éthique de la recherche en ligne qui, par ignorance ou par commodité, ont tendance à transposer aux espaces numériques des catégories élaborées pour des espaces physiques. En cela, ces approches normatives se font l'écho d'une littérature portant sur l'éthique de la recherche en ligne qui invite les chercheurs à déterminer a priori le caractère soit privé soit public de leurs données, indépendamment du statut que les acteurs concernés confèrent à leurs pratiques (Latzko-Toth et Proulx, 2013). Or, nous avançons que ces catégories - et en particulier la dichotomie public/privé - sont inadéquates pour penser les pratiques numériques et les enjeux relatifs au bien-être des personnes impliquées dans la recherche. Nous proposons plutôt de considérer le degré de publicité des données recueillies, et soutenons que l'essentiel, par respect des personnes impliquées dans la recherche, est de nous préoccuper des torts que l'amplification de cette publicité peut leur causer, qu'elle soit le fait des chercheurs, des acteurs impliqués, ou de tiers. Notre ambition est avant tout pratique: nous n'avons pas la prétention de renouveler radicalement les perspectives théoriques ou l'outillage conceptuel des chercheurs qui réfléchissent sur la nature de toutes les zones d'ombre des pratiques se trouvant à mi-chemin entre privé et public et qui sont parfois l'objet de nos

\footnotetext{
${ }^{1}$ Les auteurs tiennent à remercier les évaluateurs anonymes, ainsi que Serge Proulx, pour leurs commentaires pertinents sur une version antérieure de cet article. Nous adressons aussi de chaleureux remerciements à Hélène Bourdeloie pour ses relectures attentives et ses encouragements à toutes les étapes de ce projet d'écriture.
} 
Par-delà la dichotomie public/privé :

la mise en visibilité des pratiques numériques et ses enjeux éthiques

recherches. De façon plus pragmatique, nous souhaitons plutôt développer une réflexion visant à éclairer spécifiquement les enjeux éthiques de la recherche dans les mondes numériques en regard de ce que serait le caractère privé ou public des données recueillies dans ces contextes, afin de proposer un cadre conceptuel commun pour penser les pratiques des acteurs et des chercheurs. En outre, notre ambition n'est pas non plus normative. Évoluant dans un cadre institutionnel, celui des universités canadiennes, où nous sommes déjà soumis à un ensemble contraignant de procédures et de dispositifs de contrôle éthique et alors que ces procédures et dispositifs tendent plutôt à se développer et à se multiplier qu'à se raréfier, il nous paraît urgent de développer une réflexion, appuyée sur notre expérience de recherche sur les pratiques numériques, qui puisse contribuer à nourrir les réflexions et discussions des praticiens, éthiciens, chercheurs et administrateurs qui œuvrent à définir les cadres en question.

\section{Les apories de la dichotomie public/privé appliquée aux pratiques numériques}

La nécessité de protéger la vie privée des personnes découle d'un principe éthique fondamental : le respect de la dignité humaine. Or, tant les chercheurs que les experts chargés d'évaluer la conformité des protocoles d'enquête à ce principe $^{2}$ sont confrontés à l'ambivalence des espaces de communication numériques en ce qui a trait à la vie privée. Une ambivalence que l'on retrouve dans les pratiques et attitudes paradoxales des usagers d'Internet (Barnes, 2006 ; Rallet et Rochelandet, 2011) : d'un côté, ils semblent exposer leur vie privée en toute insouciance sur les réseaux socionumériques - prenant peu de précautions, par exemple, pour sécuriser leurs profils Facebook sur lesquels ils livrent beaucoup de renseignements personnels - et de l'autre, ils sont scandalisés d'apprendre que des organisations gouvernementales ou commerciales ont accès à ces informations, ou que leur vie privée a été exposée à d'éventuels regards indiscrets à la suite d'un changement de configuration ou d'une défaillance technique. Les choses se compliquent dans la mesure où la pratique devient courante sur les médias sociaux de tenir des conversations privées en public (Cardon, 2012). Enfin, en raison de la «recherchabilité » des contenus textuels - et même désormais visuels ${ }^{3}$ -

\footnotetext{
${ }^{2}$ Encore peu implantés en France, ces comités sont généralisés aux États-Unis et au Canada (voir Latzko-Toth et Proulx, 2013).

${ }^{3}$ La fonction "Search by Image " de Google permet, comme son nom l'indique, d'effectuer une recherche sur le web à partir d'une image. Un service similaire (Imideo) développé par une compagnie coréenne permet une recherche de fichiers vidéo à partir d'une image fixe (Pisani, 2012).
} 


\section{Guillaume LATZKO-TOTH et Madeleine PASTINELLI}

publiés sur le web et de la possibilité de recouper les données (Zimmer, 2010), il est devenu pratiquement inopérant de décontextualiser des éléments de discours pour en protéger les auteurs. Plus encore, des études récentes ont démontré que des algorithmes pouvaient assez facilement identifier de façon très précise des individus à partir des traces numériques qu'ils laissent derrière eux, laissant entrevoir la quasi-impossibilité, à terme, d'être anonyme sur Internet (Montjoye et al., 2013). Enfin, le recours aux définitions juridiques de la frontière public/privé n'est pas plus éclairant. Tandis que la justice française a conclu en avril 2013 que la publication d'un statut sur une page Facebook était d'ordre privé, l'autorité américaine des marchés financiers a considéré au même moment qu'il s'agissait au contraire d'une forme de communication publique. En effet, en juillet 2012, le PDG de l'entreprise américaine Netflix a annoncé sur sa page Facebook que son service de vidéo à la demande via Internet avait franchi le cap du milliard de visionnements mensuels. La Securities and Exchange Commission (SEC), agence gouvernementale des États-Unis surveillant les transactions financières, lui a d'abord reproché de n'avoir pas diffusé l'information suffisamment publiquement, avant de conclure en avril 2013 que la dissémination d'une information financière sur "les médias sociaux » relevait bien de la diffusion publique (voir SEC, 2013). En revanche, dans une affaire française opposant une femme à son ancien employeur (qui l'avait licenciée pour avoir critiqué «publiquement » sa supérieure sur sa page Facebook), les juges ont statué que les propos n'avaient pas été tenus « en public » et que la page en question constituait un espace privé, dans la mesure où elle est accessible à un nombre restreint d'individus choisis, formant "communauté d'intérêt » (Gradt, 2013). En somme, tout se passe comme si l'essor des médias numériques rendait plus manifeste l'existence d'espaces au statut intermédiaire, pseudo-privé ou quasi public ; un entre-deux sur le mode du "clair-obscur " (Cardon, 2008).

Dans ce contexte, comment le chercheur en sciences sociales est-il censé agir pour s'assurer de ne pas nuire aux individus impliqués de près ou de loin dans sa recherche? Est-il possible d'observer les pratiques numériques des individus tout en protégeant leur vie privée ? Et comment départager ce qui en relève de ce qui n'en relève pas dans les matériaux analysés ? À l'analyse, il nous apparaît que les perspectives adoptées et les principes mis en œuvre pour résoudre ce problème conduisent souvent à des impasses.

Un premier écueil surgit dès lors que l'on veut rattacher le caractère public ou privé des données à leur accessibilité. La notion d'accessibilité correspond à des représentations spatiales ou topologiques, lesquelles invitent à concevoir le statut privé ou public comme une propriété d'un lieu, domaine ou espace où seraient situées les pratiques étudiées. C'est par analogie avec des lieux 
Par-delà la dichotomie public/privé :

la mise en visibilité des pratiques numériques et ses enjeux éthiques

sociaux comme la rue ou la chambre à coucher que l'on détermine si l'espace étudié est public ou privé. On est ainsi amenés à conclure que c'est la procédure d'authentification et le mot de passe - protégeant l'accès à l'espace virtuel comme la serrure et la clé protègent l'accès au lieu physique-, qui permettent de distinguer les contenus privés. Amy Bruckman (2002) fut parmi les premières à suggérer que l'information en ligne pouvait être analysée sans restriction dans la mesure où aucun mot de passe n'était requis pour y accéder. $A$ priori, cela semble raisonnable, dans la mesure où l'on protège des informations privées avec des mots de passe - c'est le cas de notre compte de courriel ou de notre profil Facebook. Mais c'est aussi le cas des profils que publient les usagers des sites web de rencontres comme Meetic ou Match.com. N'importe qui, à tout moment, peut créer un compte et consulter ces profils, mais il faut pour cela détenir un compte et donc saisir un mot de passe. C'est également le cas de nombreux espaces d'interaction en ligne où le mot de passe ne sert pas à limiter l'accès à ses interventions (pour cela, on utilisera plutôt des modalités d'échange interindividuelles comme la messagerie instantanée ou le message dit " privé "); il sert plutôt à authentifier son identité, à s'assurer que personne d'autre ne va pouvoir se brancher sous son pseudonyme et se faire passer pour soi afin, par exemple, d'écrire publiquement en son nom. Si l'on ajoute que les procédures d'authentification sont parfois imposées sur des plateformes parce qu'elles constituent un moyen pour les opérateurs d'effectuer un suivi des activités des usagers et de monnayer les données ainsi recueillies, le lien entre domaine privé et mot de passe apparaît encore moins évident.

Une approche diamétralement opposée consiste à considérer que la définition des contours de la vie privée devrait être laissée aux acteurs et que les chercheurs, plutôt que de se fonder sur une définition essentialiste de ce qui constitue une interaction privée, devraient plutôt se fier aux attentes (expectations) des personnes, une notion qui a notamment été discutée par Roberts, Smith et Pollock (2004) et qui se trouve au cœur de l'idée, défendue par plusieurs auteurs (King, 1996 ; Barnes, 2004 ; Sveningsson, 2008), d'une privacité «perçue " (perceived privacy). C'est encore cette même idée que l'on rencontre, dans l'Énoncé de politique d'éthique de la recherche des trois Conseils au Canada (EPTC $2^{4}$ ). Le document stipule que les chercheurs n'ont pas à soumettre leur projet de recherche à l'évaluation par un comité d'éthique

\footnotetext{
${ }^{4}$ Après plusieurs années de mise en application, la première version de ce texte officiel, qui datait de 1998, a été revue et une seconde version a été adoptée en 2010 ; c'est pourquoi le sigle est désormais suivi du nombre 2. Le document est disponible à l'adresse suivante : http://www.pre.ethics.gc.ca/fra/policy-politique/initiatives/tcps2-eptc2/Default/.
} 


\section{Guillaume LATZKO-TOTH et Madeleine PASTINELLI}

ni à obtenir le consentement d'éventuels «participants " s'ils travaillent à partir d'informations accessibles au public et "s'il n'y a pas d'attente raisonnable en matière de vie privée " liée à cette information. Cette approche pragmatiste, qui a le mérite de respecter le point de vue des acteurs et le sens qu'ils donnent à leurs pratiques, soulève plusieurs difficultés. À commencer par celle de son opérationnalisation. En effet, les perceptions varient d'un individu à l'autre, ne serait-ce que parce que les représentations des dispositifs sont liées à la diversité des degrés et des formes d'appropriation des technologies numériques (voir Millerand, 2003). Par ailleurs, ces « attentes" sont susceptibles aussi d'évoluer chez un même individu, à différents moments, par exemple, en réalisant après coup les conséquences possibles d'une publication, ce qui explique les retraits, modifications, etc. Une façon de contourner cette difficulté consiste à postuler l'existence de normes tacites socialement partagées par les usagers d'un dispositif ou d'un espace d'interaction et de proposer, avec Helen Nissenbaum (2004), que la frontière public/privé dépendrait des règles du jeu social propres à chaque contexte particulier de communication. C'est sur cette idée, voulant qu'il soit nécessaire de tenir compte des normes de partage de l'information communément partagées par les acteurs, que repose le principe du respect de "l'intégrité contextuelle » (ibid.) des données, idée sur laquelle nous reviendrons dans la dernière section de cet article.

Un argument souvent invoqué par les partisans de l'approche fondée sur les attentes des acteurs est qu'il arrive que ceux-ci n'aient pas conscience de s'exprimer en public. Pire, que cette confusion serait induite par le dispositif luimême qui, dans le cas du web en particulier, ne permet pas de prendre la mesure de l'audience réelle - contrairement par exemple aux salons de chat, dans lesquels les autres usagers présents sont "matérialisés » par un avatar ou encore inciterait à se livrer davantage qu'on ne le ferait dans un cadre physique, en face à face (Walther, 1996 ; Barnes, 2004). S'il est en effet indiscutable qu'il est bien souvent difficile, sinon impossible, d'avoir une idée de l'audience actuelle et future de ses interventions, plusieurs indices permettent de croire que les usagers des espaces d'interaction en ligne (forums de discussion, chats, mondes virtuels, etc.) sont néanmoins bien conscients du fait que leurs interventions seront accessibles à un nombre indéterminé (voire potentiellement illimité) de regards dès lors qu'elles prennent place dans l'espace d'échange commun. Dans ce sens, il faut peut-être rappeler que le recours au pseudonyme, s'il vise parfois à s'assurer que l'on ne pourra être retracé hors ligne par ceux avec qui on échange en ligne, permet aussi - et peut-être avant tout - de s'assurer qu'on ne sera pas identifiable dans le cas, peu probable mais possible, où un voisin ou un collègue devait un jour tomber sur les traces engendrées par ces pratiques numériques. II est de même très courant (et socialement acceptable) d'éviter de révéler certaines informations 
Par-delà la dichotomie public/privé :

la mise en visibilité des pratiques numériques et ses enjeux éthiques

qui nous rendraient trop facilement identifiables ou d'afficher une photographie de soi sur son profil d'utilisateur. Si les internautes avaient réellement des attentes en matière de vie privée et se croyaient à l'abri des regards, pourquoi prendraient-ils ces précautions ? Les observations menées par Pastinelli (2007) auprès de membres d'une communauté en ligne ont révélé des pratiques de communication sophistiquées, alternant subtilement les interactions à la vue de tous («publiques») ou au contraire à l'abri des regards («privées»). Or, l'analyse des interactions montre bien que les échanges qui ont lieu en privé, par courriel, par messagerie instantanée ou autrement, sont bien différents de ceux, consultables par tous, qui ont lieu dans l'espace d'échange collectif. En somme, aussi intimes que puissent parfois être les questions abordées, les participants à ces espaces accessibles à tous ne s'y comportent pas comme s'ils se trouvaient dans un espace privé. Et on peut croire qu'il en est de même dans tous les contextes sociaux numériques où les échanges consultables par tous donnent lieu en parallèle à des échanges qui ne sont accessibles qu'à un nombre restreint d'interlocuteurs choisis.

Enfin, une troisième source de confusion provient de l'équation posée entre « privé » et « intime ». De là à conclure que les contenus à caractère intime relèvent nécessairement de la vie privée et qu'ils devraient donc être sujets aux dispositions liées au respect de la vie privée, il n'y a qu'un pas que certains auteurs n'hésitent pas à franchir (voir notamment McKee et Porter, 2009). Or il s'agit pourtant de deux notions bien distinctes. L'intimité se conçoit par opposition à l'extériorité (Ricoeur, 1988) ; elle est ce qui, de l'expérience et de l'identité de l'individu, n'est pas visible ou saisissable de l'extérieur, par autrui, et donc qui appartient en propre à la subjectivité individuelle. Par extension, on considère couramment comme «intimes » les relations dans lesquelles on est amené à mettre en mots son intimité pour la partager avec d'autres, le plus souvent des proches, mais pas nécessairement. La vie privée, quant à elle, se définit plutôt par opposition avec la vie publique, soit cette part de notre vie qui, si elle n'est pas nécessairement d'intérêt public, est portée à la connaissance de tous. L'opposition entre vie privée et vie publique renvoie en fin de compte au contrôle qu'exerce l'individu sur son image. On considère ainsi comme publique une information connue de tous ou, plus exactement, connaissable de tous, parce que disponible et accessible à n'importe qui. À l'inverse, on considère comme privée une information qui n'est pas connaissable de tous et que l'individu est libre de voiler ou dévoiler comme bon lui semble, au gré des circonstances, mais toujours de façon contrôlée (Tisseron, 2011). Par nature, l'intimité relève a priori de la vie privée, mais l'individu concerné peut choisir de la rendre publique, en l'exposant à la télévision (Mehl, 1996), dans un blogue (Rouquette, 2008), ou dans les pages d'un grand quotidien. Un cas récent, 


\section{Guillaume LATZKO-TOTH et Madeleine PASTINELLI}

emblématique, est la lettre ouverte d'Angelina Jolie au New York Times, dans laquelle elle évoquait la double mastectomie préventive qu'elle venait de subir (Jolie, 2013). Est-il sujet plus intime que la crainte de la mort et le rapport à son corps qui était au cœur de ce qu'écrivait l'actrice ? Et peut-on concevoir publicité plus grande que celle obtenue par la reprise de cette lettre dans les médias du monde entier en raison de l'extrême célébrité de son auteure ? Ce n'est donc pas le caractère intime des informations qui fait que celles-ci peuvent porter atteinte à la vie privée, c'est le fait qu'elles ne soient pas accessibles à tous et qu'elles le deviennent.

Respecter la vie privée d'un individu, c'est respecter son droit de contrôler l'accès à ce qui, de sa vie, n'est pas déjà public. Or, nous avons vu que dans la plupart des cas, les traces d'activité recueillies sur Internet sont de nature publique. Les problèmes qui peuvent surgir ne sont donc pas liés à la frontière public/privé. Conséquemment, la pertinence de cette dichotomie comme outil analytique pour fonder les décisions des chercheurs sur un plan éthique est discutable, et il nous apparaît nécessaire de lui substituer un autre cadre d'analyse.

\section{La recherche en ligne est-elle soluble dans l'éthique?}

Pour circonscrire les enjeux éthiques de la recherche en ligne, il convient d'abord de clarifier le rapport entre ces deux principes éthiques que sont le respect de la vie privée et la nécessité d'obtenir le consentement des participants à la recherche ${ }^{5}$, c'est-à-dire des personnes auprès desquelles le chercheur fait son enquête. On considère habituellement qu'un chercheur qui travaille exclusivement sur des données publiques, accessibles sans restriction, peut développer son analyse et publier ses travaux sans s'inquiéter d'obtenir au préalable le consentement libre et éclairé des acteurs concernés et cela même dans le cas où ils sont identifiables. On pense ici par exemple aux chercheurs s'intéressant aux discours et pratiques observables dans une émission de téléréalité (Ehrenberg, 1995 ; Jost, 2002, 2009), aux propos tenus par les participants d'une ligne ouverte à la radio (Vincent, Turbide et Laforest, 2008) ou encore aux discours publics d'hommes et de femmes politiques (Martel, 2012). Or, on peut se demander en vertu de quel principe le caractère public des données utilisées par le chercheur permet de passer outre la nécessité d'obtenir le consentement des personnes sur lesquelles porte la recherche. Est-ce parce

\footnotetext{
${ }^{5}$ Nous distinguons ici les personnes qui participent à la recherche de celles qui en sont l'objet sans pour autant y participer.
} 
Par-delà la dichotomie public/privé :

la mise en visibilité des pratiques numériques et ses enjeux éthiques

que l'on considère que travailler sur des émissions télévisées, des articles de presse ou des billets de blogue consiste à étudier des artefacts culturels plutôt que des personnes? Ou parce que les données publiques sont le fait de personnes elles-mêmes publiques qui, dès lors qu'elles s'expriment au nom de la fonction que leur confère ce statut, peuvent faire l'objet d'un traitement particulier? Si on peut aisément écarter ces deux premières pistes de réponse, il en est deux autres entre lesquelles il nous apparaît a priori plus difficile de trancher. En effet, la question du consentement n'aurait-elle finalement pour raison d'être que la nécessité de protéger la vie privée des personnes ? Ou ne serait-ce pas plutôt parce que l'objet du consentement est le fait même de participer à la recherche, c'est-à-dire d'accorder au chercheur un accès privilégié à certaines informations personnelles, auquel cas on ne saurait considérer comme des "participants" (et donc se préoccuper de leur consentement) ceux qui n'accordent au chercheur aucun accès privilégié à des informations les concernant?

Dans ce sens, il nous semble que, dans plusieurs réflexions sur les enjeux éthiques de la recherche en ligne, on confond parfois le consentement à participer à la recherche avec le consentement à faire l'objet d'un discours savant. II peut arriver que des individus, qui ne s'y attendaient pas, découvrent après coup que des chercheurs s'étaient penchés sur des contenus qu'ils avaient publiés en ligne et qu'ils éprouvent alors un malaise, qu'ils soient choqués et aient l'impression qu'on viole leur vie privée, faisant ainsi part de leur désapprobation aux chercheurs en question ${ }^{6}$. Dans la mesure où il est question de recherche ne portant que sur des contenus accessibles à toute personne ayant accès à Internet ${ }^{7}$, sont-ce bien les attentes ${ }^{8}$ en matière de vie privée qui

\footnotetext{
${ }^{6}$ Peu de cas ont été documentés mais cette situation est régulièrement évoquée sous forme de cas hypothétique (voir par exemple: King, 1996). Des cas réels où des usagers de forums de soutien mutuel entre personnes atteintes du cancer se plaignent de se savoir observées par des chercheurs sont rapportés par Eysenbach et Till (2001).

${ }^{7}$ II va sans dire qu'il faut considérer autrement les messages échangés en privé, par courriel, via des médias sociaux ou d'autres plateformes. Nous y reviendrons.

${ }^{8}$ Dans l'EPTC 2, on emploie la formule "attente raisonnable en matière de vie privée " comme équivalent de "reasonable expectation of privacy». Cette notion est loin d'être limpide. En effet, que considère-t-on comme étant des "attentes " et dans quelle mesure ce que l'on désigne ainsi n'est-il pas formulé uniquement a posteriori par celui qui est agacé par la démarche du chercheur? Plus encore, comment tracer la limite entre des attentes qui seraient raisonnables et d'autres qui ne le seraient pas ? Et comment considérer comme "raisonnable " l'attente que ne devienne pas public un contenu que l'on diffuse dans un espace que l'on sait être accessible et consultable par n'importe qui ? II nous semble par ailleurs essentiel de souligner le fait que les « attentes » les plus largement partagées quant à la visibilité des contenus et aux conséquences éventuelles de cette visibilité ont certainement changé au fil des ans, alors que la question des risques d'atteinte à la vie privée découlant de certains usages d'Internet a fréquemment été abordée dans les médias de masse. II est bien sûr à prévoir que ces attentes continueront de changer dans l'avenir, à mesure
} 


\section{Guillaume LATZKO-TOTH et Madeleine PASTINELLI}

sont en jeu ou ne serait-ce pas plutôt le consentement à être l'objet d'une démarche de recherche ? II nous paraît essentiel de bien distinguer les deux. Si l'on peut se soucier du consentement des participants pour maintes raisons et notamment parce que la recherche peut supposer une incursion du chercheur dans l'espace de la vie privée, il n'en demeure pas moins que ces deux questions ne sont pas réductibles l'une à l'autre. On peut en effet très bien imaginer quantité d'entreprises de recherche portant exclusivement sur des documents publics, n'impliquant aucune atteinte à la vie privée, mais qui seraient néanmoins susceptibles de déplaire fortement à ceux qui en sont l'objet. On pense par exemple à des démarches d'analyse de contenu prenant pour objet les écrits et les propos tenus en public par certains élus et visant à mettre en lumière leurs aprioris idéologiques. Si l'on devait au préalable obtenir le consentement à être l'objet de la recherche des personnes concernées, il y a fort à parier que de telles recherches ne pourraient jamais être entreprises. De la même manière, il faut rappeler que, même lorsqu'ils ont consenti à participer à la recherche, les sujets qui prennent part à nos enquêtes ne sont ensuite pas toujours disposés à se reconnaître dans nos analyses ni à les endosser. Mais cela n'implique pas nécessairement que le travail porte atteinte à leur vie privée ou que les matériaux utilisés aient été l'objet d'«attentes » en matière de vie privée.

Au-delà de la surprise ressentie par des individus devant l'intérêt de chercheurs pour certains des discours qu'ils tiennent publiquement sur Internet et qu'ils n'auraient jamais imaginés a priori susceptibles d'être abordés d'une telle manière ${ }^{9}$, l'éventuelle irritation éprouvée par un individu dans pareille circonstance est susceptible d'être avant tout nourrie par la crainte que l'analyse du chercheur ne débouche sur une image de soi peu flatteuse, dans laquelle on ne se reconnaît pas ou encore qui ne donne à certaines de ses pratiques un sens qui s'avère en rupture avec celui qu'on leur donne soi-même habituellement. Pour le dire plus simplement, il nous semble que ce qui est en jeu, c'est le fait que les acteurs soient potentiellement en désaccord avec un discours savant sur lequel ils n'ont aucun contrôle ou qu'ils n'aient tout simplement aucune envie que les discours qu'ils tiennent en public soient l'objet d'une analyse savante.

Or, cette confusion nous semble dangereuse et il nous paraît impératif d'insister pour que l'on distingue clairement le fait de participer à la recherche et

que plus de gens sa familiariseront avec les enjeux relatifs à différents dispositifs numériques, en évolution constante.

9 Et il convient ici de souligner que les participants d'une ligne ouverte ou d'une émission de téléréalité, voyant leur image ou leurs propos utilisés dans le cadre d'un travail de recherche, risquent d'éprouver exactement le même sentiment de surprise et d'étonnement. 
Par-delà la dichotomie public/privé :

la mise en visibilité des pratiques numériques et ses enjeux éthiques

le fait d'être l'objet d'un discours savant. L'amalgame de ces registres et le zèle vertueux sur lequel débouche parfois la crainte de négliger des risques éthiques nous semblent susceptibles de mener vers deux dérives aussi redoutables l'une que l'autre. La première serait d'ériger en obligation éthique le fait, pour les chercheurs, d'obtenir le consentement non pas uniquement de ceux qui participent à la recherche, c'est-à-dire qui donnent au chercheur un accès privilégié à certaines données ou informations les concernant, mais bien plus largement de ceux qui sont l'objet d'un discours du chercheur alors même qu'ils ne participent pas à la recherche. Cela voudrait dire que les chercheurs ne pourraient plus réfléchir et développer d'analyse sur ce qui se joue dans l'espace public, à moins d'y être préalablement autorisés par ceux qui prennent la parole en public. La seconde dérive consisterait plus radicalement encore à confondre le consentement à participer à la recherche avec le fait de se reconnaître dans l'analyse produite par le chercheur et de l'avaliser.

Ce dernier risque n'est pas aussi exagéré qu'il y paraît de prime abord. Dans une certaine perspective culturaliste, on n'hésite pas à considérer que seuls les acteurs eux-mêmes (ou les membres de leur groupe ou leurs représentants autoproclamés) sont en position pleinement légitime pour tenir un discours sur leur univers social et leurs pratiques, ce qui n'est pas loin par moments de déboucher sur l'obligation, pour le chercheur, de soumettre avant publication ses analyses à ceux qui en sont l'objet et de s'abstenir de faire de la recherche sur une question ou de développer une analyse dès lors que celle-ci ne convient pas à ceux dont il est question. Dans le contexte canadien, l'application qui est faite par les comités d'éthique de la recherche (CER) de l'EPTC 2 dans le cas des enquêtes menées en contexte autochtone a rendu extrêmement difficile, sinon impossible, la poursuite de recherches ou la publication de travaux susceptibles de déplaire aux autorités en place. En effet, les règles prévoient que la recherche effectuée en milieu autochtone soit menée en collaboration avec les communautés concernées et avec leur accord, le chercheur devant tenir compte du «point de vue autochtone ${ }^{10}$ »: un point de vue que l'on présuppose relativement homogène et, dans tous les cas, différent de celui du chercheur, s'il n'est pas lui-même autochtone. Le consentement individuel de ceux qui participent à la recherche apparaît comme insuffisant, les chercheurs devant également soumettre leur projet de recherche pour approbation aux "communautés ${ }^{11}$ », c'est-à-dire concrètement aux leaders politiques en place.

\footnotetext{
${ }^{10}$ L'EPTC 2 emploie plus exactement les notions de conception du monde, de culture et d'identité. Nous résumons ici l'idée avec la notion de point de vue autochtone.

${ }^{11}$ La notion de communauté est centrale dans le chapitre de l'EPTC 2 consacré à la recherche en milieu autochtone, lequel définit la communauté comme une « collectivité qui partage une identité ou des intérêts et qui a la capacité d'agir ou de s'exprimer en tant que groupe » (p. 119).
} 


\section{Guillaume LATZKO-TOTH et Madeleine PASTINELLI}

Les chefs autochtones disposent donc de facto d'un redoutable droit de veto sur les travaux des chercheurs. L'EPTC 2 prévoit clairement que l'obligation pour les chercheurs de travailler en collaboration avec les communautés ne devrait pas empêcher le développement de perspectives critiques de la part des chercheurs. Le texte précise en outre qu'il n'y a pas lieu d'assimiler les autorités en place à la "communauté » et qu'on peut obtenir l'appui et la collaboration de la communauté autrement qu'en sollicitant des autorisations des conseils de bandes. Mais en pratique, il semble que les CER tendent à faire des interprétations extrêmement précautionneuses de l'EPTC 2 et encouragent toujours, de façon assez systématique, les chercheurs à solliciter l'approbation des autorités autochtones.

Cette tendance est très préjudiciable du point de vue de l'intérêt commun, en ce qu'elle rend à peu près impossible pour les chercheurs l'adoption de perspectives critiques. Plus fondamentalement encore, s'il faut considérer que le travail produit par le chercheur n'est acceptable que dans la mesure où il coïncide avec l'image que veulent donner ou qu'ont d'eux-mêmes, de leur univers et de leurs pratiques ceux qui en sont l'objet, on risque à terme de condamner les sciences sociales à la plus complète insignifiance ${ }^{12}$. En effet, si seuls les acteurs eux-mêmes ou les représentants autoproclamés ou élus de leur groupe sont en posture légitime pour tenir un discours sur leur monde et ce qu'ils y font ou pour savoir quelles interprétations doivent être considérées comme vraies ou valables, quelle est alors l'utilité des sciences sociales ? Si tout ce que peut faire le chercheur est de tendre à ceux qui sont l'objet de la recherche un miroir dans lequel ils pourront se retrouver exactement tels qu'ils se sont énoncés ou d'agir comme porte-parole de ceux auprès desquels il enquête, le chercheur apparaît alors comme un intermédiaire aussi superflu qu'encombrant. À ce compte-là, ne devrions-nous pas plutôt fournir aux acteurs des tribunes d'où ils pourront énoncer eux-mêmes et sans intermédiaire la réalité de leur univers social et de leurs pratiques?

Or, il nous semble raisonnable de croire que le rôle du chercheur n'est pas simplement de relayer le discours des acteurs et il est réaliste d'envisager que des analyses justes, pertinentes et éclairantes peuvent parfois être mal reçues par ceux-là mêmes qui en sont l'objet. Sans défendre l'idée que la seule connaissance pertinente serait celle reposant sur une coupure épistémologique permettant de se libérer de ce que seraient les obstacles épistémologiques (au sens de Bachelard) de la connaissance ordinaire, de telle sorte que les acteurs concernés seraient a priori comme par nature toujours incapables de voir clair

\footnotetext{
${ }^{12}$ Pour une critique systématique des apories de la perspective culturaliste, nous invitons le lecteur à se référer aux écrits lumineux de l'ethnologue Jean Bazin, et plus particulièrement à son article intitulé «Question de sens » (Bazin, 1998).
} 
Par-delà la dichotomie public/privé :

la mise en visibilité des pratiques numériques et ses enjeux éthiques

sur leurs propres pratiques, il nous paraît essentiel de reconnaître que même dans une démarche s'en tenant à une description empirique ou dans le cadre d'une entreprise de type compréhensif qui vise à restituer l'expérience des acteurs, le point de vue du chercheur de terrain n'a pas à être assimilable à celui des acteurs eux-mêmes. D'abord, il convient de souligner que les acteurs peuvent parfois, et pour une quantité de raisons différentes, ne pas avoir intérêt (économiquement, politiquement ou socialement, voire psychologiquement) à reconnaître comme juste une analyse qu'ils savent pourtant coller de près à leurs expériences, leurs pratiques et leurs discours. Outre ces situations, il faut rappeler plus fondamentalement encore qu'une description n'est jamais simplement la saisie directe puis le codage d'un réel objectif. Comme le disait si bien Lenclud « on ne peut voir que ce qu'on regarde » (Lenclud, 1995) et le regard lui-même n'est jamais affranchi des catégories de langage ou de sens. II dépend en outre du «point de vue » de l'observateur (voir aussi Laplantine, 1996), de telle sorte que décrire c'est toujours déjà interpréter. Et bien entendu, les questions que se pose le chercheur, les catégories à partir desquelles il problématise les phénomènes qui l'intéressent et donc le point de vue qui est le sien ont toutes les chances de ne pas être assimilables au point de vue des acteurs eux-mêmes qui, eux, sont pris dans l'action et ont des préoccupations d'une autre nature.

En somme, s'il est courant pour un chercheur de s'intéresser au sens que donnent les acteurs à leurs pratiques et aux interprétations qu'ils font euxmêmes de leur univers, on peut aussi avoir d'excellentes raisons de considérer que le «point de vue de l'acteur» est loin d'épuiser le registre des interprétations possibles, voire que d'autres interprétations sont nécessaires pour bien comprendre le phénomène étudié. On peut d'ailleurs considérer que le «point de vue de l'acteur» est en lui-même une donnée qui doit être analysée et mise en perspective. Comme Jean Bazin le soulignait avec insistance, le discours que tiennent des individus sur leurs pratiques obéit luimême à un ensemble de règles et dépend toujours de son contexte d'énonciation. En fin de compte, tenir un discours sur l'action «c'est une autre action, ce n'est pas le sens de la première »(Bazin, 1998, p. 33). Outre les rapports de force et de sens qui sont en jeu dans la production d'une image de soi à l'intention de ses interlocuteurs et éventuellement du chercheur, on peut aussi souligner que ce problème d'épistémologie nous mène ultimement toujours à la question de la conscience et de l'éventuelle transparence ou opacité du soi à lui-même. Giddens (1984) juge ainsi utile de distinguer, d'une part, la conscience pratique qu'il qualifie de «non consciente » et qui recouvre tout ce que les acteurs savent faire en pratique) et, de l'autre, la conscience discursive qui, elle, renvoie à tout ce que les acteurs savent exprimer, aux 


\section{Guillaume LATZKO-TOTH et Madeleine PASTINELLI}

discours qu'ils peuvent tenir. C'est exactement de la même manière que Bourdieu (1980), dans sa théorie de la pratique, s'applique à mettre en évidence le fait que les individus agissent couramment par "sens du jeu », c'est-à-dire sans être en mesure d'expliciter l'ensemble des règles auxquelles ils savent pourtant très bien obéir en pratique. L'enquête de terrain est ainsi couramment l'occasion de constater que les participants à la recherche, même avec la meilleure foi du monde et un désir sincère et réel d'aider le chercheur, ne sont pas toujours en mesure de décrire correctement leurs pratiques, que ce qu'ils en disent est parfois fort éloigné de ce qu'on peut observer ou de ce qu'ils racontent à d'autres moments; que leur discours est loin d'être toujours univoque et peut parfois s'avérer porteur de contradictions qu'on ne saurait ignorer ou gommer.

Sans nous attarder plus longuement sur une question d'épistémologie qu'on ne saurait épuiser dans le cadre de cet article, on peut en outre souligner qu'il existe une large variété de perspectives théoriques dans lesquelles il apparaît probable qu'au terme de l'enquête, le chercheur soit amené à produire une analyse qui excède la compréhension qu'ont les acteurs eux-mêmes de leur expérience, voire qui puisse consister en une complète déconstruction de cette compréhension des choses. C'est le cas d'à peu près toutes les approches relevant du marxisme, de la psychanalyse ou du structuralisme, mais c'est également vrai de cadres théoriques proposés plus récemment par des auteurs comme Giddens (1984), Lahire (1998, 2013), Kaufmann (2001), Martuccelli (2002) ou Lemieux (2009).

Dans la mesure où il y a potentiellement un décalage entre l'analyse du chercheur et la compréhension qu'ont de leur monde et de leurs pratiques les acteurs eux-mêmes, on conçoit bien que des gens puissent ne pas avoir envie d'être l'objet de la recherche et éventuellement qu'ils protestent devant l'entreprise du chercheur. Cependant, le respect de la vie privée des personnes et l'obligation d'obtenir le consentement libre et éclairé de ceux qui participent à la recherche n'impliquent pas que le chercheur doive obtenir l'autorisation des acteurs pour se pencher sur ce qu'ils font et disent publiquement et dont n'importe qui, au même titre que le chercheur, peut prendre connaissance. En revanche, cela ne veut pas dire pour autant que le chercheur soit exonéré de la responsabilité de veiller au bien-être des personnes qui sont l'objet de la recherche et, surtout, de se soucier d'éviter de leur nuire. Et sur ce plan, la question qui se pose n'est ni celle de l'accessibilité des informations, ni celle des attentes des acteurs, mais bien plutôt celle du degré de publicité des informations et de l'effet qu'est susceptible d'avoir l'intervention du chercheur sur cette publicité. 
Par-delà la dichotomie public/privé :

la mise en visibilité des pratiques numériques et ses enjeux éthiques

\section{La publicité des pratiques et ses transformations}

Classiquement, les chercheurs sur les usages des technologies de l'information et de la communication $(T I C)^{13}$ ont examiné deux sortes de pratiques sociotechniques en lien avec les médias numériques. D'un côté, des pratiques individuelles privées, généralement en contexte domestique, mais aussi professionnel, et captées in situ ou dans des récits de pratique ; de l'autre, des pratiques d'audiences au sens que l'ethnographie des médias donne à ce terme (voir Livingstone, 2004), c'est-à-dire des manifestations médiatisées de participation à des collectifs d'usagers réunis dans leur usage commun d'un même média numérique ${ }^{14}$. Sous l'influence d'un lourd héritage théorique l'opposant à la notion de public, la notion d'audience a habituellement été associée à la sphère privée (Dayan, 2000). II s'ensuit une grande difficulté à penser les pratiques numériques autrement que privées jusqu'à preuve du contraire, avec tous les dilemmes cornéliens que cela entraîne pour les chercheurs. Et ce, alors même que les traces et contenus découlant de ces pratiques peuvent acquérir une visibilité sociale aussi grande que n'importe quel discours ou image rendus "publics" par les médias traditionnels, correspondant en cela à la dimension de monstration que Dayan (2002), avec Louis Quéré, oppose à la dimension plus politique du débat et qui ne serait pas intrinsèque à la publicité. Livingstone (2004) rappelle que l'opposition classique entre "audiences " (agrégats descriptifs conçus par des tiers) et "publics " (collectifs agissants et réflexifs), et donc entre médiatisation (ou mise en visibilité) et publicisation, a été formulée dans un contexte social, historique et intellectuel spécifique, marqué notamment par l'hégémonie des médias de masse. Nous serions entrés, depuis, dans l'ère de “l'audience diffuse ${ }^{15}$, laquelle

« ne peut plus être circonscrite dans des lieux et des moments particuliers, mais fait plutôt partie intégrante de tous les aspects de la vie quotidienne, dans les nations industrialisées en tout cas, et gagne mondialement du terrain » (Livingstone, 2004, p. 3435).

\footnotetext{
${ }^{13}$ Nous incluons ici les diverses communautés de recherche (sociologie des usages des TIC, approche de la domestication des technologies, Internet Studies, etc.) qui se sont intéressées aux pratiques numériques dans la perspective des sciences sociales.

${ }^{4}$ Un exemple typique de ce type de travaux est fourni par l'étude classique de Nancy Baym (1995) sur les membres d'un forum Usenet, qui sont eux-mêmes des téléspectatrices fans de soap operas.

${ }^{15}$ Livingstone reprend une typologie proposée par Abercrombie et Longhurst (1998).
} 


\section{Guillaume LATZKO-TOTH et Madeleine PASTINELLI}

Des audiences tous azimuts en quelque sorte, correspondant à une prolifération grandissante ainsi qu'à l'omniprésence dans la vie sociale des instances et des formes de médiatisation. À tel point que l'on peut parler d'une convergence entre audiences et publics, entre visibilité et publicité, entre le caractère médiatisé et le caractère public des pratiques. Or, à partir du moment où les pratiques que nous observons dans les espaces en ligne sont pensées et assumées comme étant publiques, les dilemmes évoqués précédemment se posent en termes nouveaux. L'enjeu le plus pressant n'est plus de savoir si une pratique (ou une parole, une trace, etc.) est publique ou pas. La question devient plutôt : comment est-elle publique ? Quelle forme prend cette publicité ? Quelles sont ses caractéristiques? Est-elle susceptible d'évoluer, de se transformer, d'être altérée d'une façon ou d'une autre? Et avec quelles conséquences?

Si les pratiques de communication en ligne semblent avoir contribué à brouiller la frontière entre privé et public, c'est notamment à cause de l'important décalage que l'on observe sur Internet entre accessibilité et visibilité des contenus. En effet, un contenu numérique peut être librement accessible et donc consultable par tous mais, en pratique, consulté par un nombre très restreint de personnes. On s'expose ainsi dans des espaces comme des forums de discussion, parfois sur des sujets «sensibles" comme la maladie, la sexualité, etc., en sachant bien qu'on peut être lu par n'importe qui, mais qu'il est peu probable que des personnes de notre entourage nous lisent. II faut cependant rappeler que le même décalage existe pour à peu près tous les contenus considérés comme publics, la notion renvoyant plutôt à la disponibilité de l'information qu'à la connaissance de celle-ci. En effet, on considère comme publiques des informations qui sont disponibles à tous (qui font la une de la presse par exemple) alors qu'en pratique, elles ne sont jamais vues et connues de tous. C'est l'ampleur de ce décalage entre accessibilité et visibilité de l'information qui s'avère inédite avec le numérique.

Les analyses de danah boyd sur les vives réactions provoquées par les changements apportés à l'interface de Facebook en 2006 contribuent à mettre en évidence l'importance de cet enjeu de la visibilité (boyd, 2006). Sur Facebook, chacun peut afficher sur son profil un certain nombre d'informations personnelles: goûts musicaux, opinions politiques, lieu de résidence, établissement scolaire, emploi, situation matrimoniale... L'usager peut à tout moment mettre son profil à jour pour qu'il reflète sa situation, par exemple en cas de rupture amoureuse. Jusqu'en septembre 2006, ces changements dans la vie des usagers étaient peu visibles, puisqu'on ne pouvait les observer qu'en visitant régulièrement le profil de l'usager en question, ce qui constitue une pratique plutôt exceptionnelle. Or, soudainement, les usagers de Facebook ont 
Par-delà la dichotomie public/privé :

la mise en visibilité des pratiques numériques et ses enjeux éthiques

découvert une nouvelle version de l'interface qui intégrait un «fil d'actualités » informant tous leurs contacts de la moindre modification apportée à leur profil : ainsi, un changement de situation amoureuse était-il désormais publicisé auprès de l'ensemble des contacts - plusieurs centaines en général ${ }^{16}$ - sous la forme d'un message du type "Untel est maintenant célibataire ». Ce fut une très mauvaise surprise pour beaucoup d'usagers, plusieurs disant avoir eu l'impression que leur intimité avait été violée par ce nouvel élément du dispositif. Or, le fil d'actualités ne fait plus débat aujourd'hui : les usagers savent ou apprennent rapidement que les mises à jour de leur profil y sont immédiatement annoncées.

L'exemple ci-dessus est d'un intérêt tout particulier pour notre propos, parce que dans ce changement apporté au dispositif, aucune information n'est devenue soudainement accessible à des gens qui n'y avaient pas accès auparavant. Seulement, au lieu d'être simplement disponible, l'information est mise en vedette, comme si l'on braquait un projecteur dessus. Ce que ce cas révèle, c'est le fait que l'on s'expose sur Internet avec en tête une certaine idée quant à ce que sera la visibilité du contenu que l'on publie, et qu'un malaise est ressenti quand ce contenu se retrouve sous un projecteur, avec une visibilité nettement plus grande que celle à laquelle on s'attendait.

Dans le cadre de cette réflexion, nous appelons «publicité " le mode d'existence d'une information ou d'un contenu médiatisé ayant minimalement un caractère public - c'est-à-dire non privé ${ }^{17}$ - et porté à l'attention d'une audience dont l'étendue est variable, mais non bornée a priori. Comme nous l'avons indiqué précédemment, la publicité d'un contenu n'est pas réductible à son accessibilité. Cette accessibilité est, en revanche, une condition nécessaire à sa visibilité (Thompson, 2000) qui, à son tour, est essentielle à la circulation de l'information ou du contenu en question et, ultimement, à l'ampleur de l'audience qui lui aura accordé son attention. La publicité d'un contenu est donc fortement corrélée à sa visibilité, mais ne lui est pas strictement équivalente ${ }^{18}$, dans la mesure où, d'une part, être visible ne suffit pas pour être remarqué, pour capter l'attention et, d'autre part, une information privée pourrait être exposée à une visibilité fortuite, involontaire, voire inconnue de la personne qui en est la

\footnotetext{
${ }^{16}$ Dans une enquête réalisée aux États-Unis en 2013, le Pew Research Center a trouvé que plus de la moitié des usagers de Facebook comptaient au moins 200 « amis » dans leur liste de contacts, la moyenne s'établissant à 338 (Smith, 2014).

${ }^{17}$ Donc non assujetti aux conditions normées et restrictives de partage de ce qui relève de la vie privée des acteurs.

${ }^{18}$ Pour une discussion de l'articulation conceptuelle entre publicité et visibilité, voir Brighenti (2010).
} 


\section{Guillaume LATZKO-TOTH et Madeleine PASTINELLI}

source $^{19}$. En revanche, la visibilité soudainement amplifiée d'un contenu accessible jusque-là obscur risque fort d'en augmenter ipso facto le degré de publicité, parce que cette mise en visibilité se traduit concrètement par un déplacement de ce contenu de son contexte d'origine (plateforme, interface, support, proximité avec d'autres contenus, nature et étendue de l'auditoire) vers un contexte radicalement différent auquel seront attachées des «normes de partage » et des attentes implicites différentes en termes de circulation.

De ce qui précède, il découle que la publicité des contenus médiatiques doit être comprise, non dans les termes d'une simple dichotomie qui opposerait deux statuts (privé ou public), mais plutôt dans les termes d'une gradation. Modalités d'accès, conditions de visibilité et circulation anticipée (en termes de nature et d'étendue du lectorat ou de l'audience) sont les trois dimensions constitutives et interreliées de ce que nous appelons le degré de publicité des informations médiatisées. Le concept s'applique aux traces numériques déposées par les internautes qui publient, commentent, qualifient ou ratifient des contenus en ligne, mais on peut l'étendre à l'ensemble des pratiques numériques, dans la mesure où les pratiques des acteurs ne sont observables en ligne que par la médiation d'une information ou d'une donnée produite dans le dispositif technique et captée par le chercheur.

L'exemple typique d'une telle amplification de la publicité est ce qui se produit quand une séquence vidéo amateur, diffusée par son auteur sur un site comme YouTube, acquiert une audience sans commune mesure avec celle anticipée au moment de sa publication initiale. L'idée d'une gradation de la publicité prend ici tout son sens puisque le contenu a toujours été public ; mais cette publicité a pris une tout autre ampleur, susceptible d'exposer la vie privée d'individus d'une manière beaucoup plus dommageable que si le contenu était demeuré obscur. Cet exemple nous permet d'attirer l'attention sur deux points essentiels. Tout d'abord, sur le fait que l'embarras, voire le sentiment de «viol » de l'intimité éprouvé par des individus dont les traces numériques sont mises en visibilité peut ne pas découler d'une invasion de leur vie privée, mais simplement d'une amplification de la publicité des traces qu'ils ont déposées sur Internet. Ensuite, cette amplification peut être le fait de toutes sortes d'acteurs : internautes ordinaires, chercheurs en sciences sociales, journalistes... En France, la presse a fait grand bruit du «portrait Google d'un inconnu », publié en novembre 2008 dans le magazine Le Tigre. Son auteur, Raphaël Meltz (2009), avait choisi un quidam sur Internet et recueilli toutes les informations publiquement accessibles sur le web à son sujet. Par divers recoupements, il avait été en mesure de divulguer des éléments de sa vie privée, jusque dans les

\footnotetext{
${ }^{19}$ Ainsi, les images de Google Street View rendent visibles des informations à caractère privé (montrant des personnes entrant ou sortant de certains lieux, par exemple).
} 
Par-delà la dichotomie public/privé :

la mise en visibilité des pratiques numériques et ses enjeux éthiques

détails de sa vie amoureuse et professionnelle, ses lieux de vacances, etc. Le cas est intéressant car il met en jeu deux mises en visibilité successives. D'abord, par mise en relation et rapprochement d'éléments issus de contextes disjoints. Puis, par l'effet de démultiplication de l'audience engendré par la "surexposition médiatique » de l'affaire (Meltz, 2009). Comme le rappelle l'auteur du texte en cause, lui-même ne pouvait imaginer que son texte publié dans un magazine indépendant à faible tirage obtiendrait une visibilité nationale, voire internationale, par le jeu des reprises et des republications dans d'autres médias.

Un autre cas, plus récent, a pour cadre la petite ville de Torrington aux ÉtatsUnis. En réaction à l'inculpation d'adolescents pour une affaire d'agression sexuelle suivie de harcèlement sur Internet (les images du viol ayant été diffusées sur Facebook), des usagers de Twitter (eux-mêmes mineurs) se sont mis à diffuser des messages d'insultes à l'endroit de la victime. Un journal local décide de dénoncer ces messages en reproduisant un échantillon de ces tweets haineux en une. Par suite, les adolescents auteurs des tweets sont à leur tour victimes de harcèlement par Internet (voir Blake, 2013). Le journal se défend en affirmant qu'il n'a fait que publier de l'information déjà publique. Or, en reproduisant sur sa une des messages extraits du flux de milliers de tweets rapidement oubliés car noyés dans la masse, il en a substantiellement transformé la publicité. L'un des aspects de cette transformation est leur décontextualisation-recontextualisation qui a pour conséquence l'altération de leur « intégrité contextuelle » (Nissenbaum, 2004).

En somme, la notion de degré de publicité des pratiques numériques permet d'éclairer la réflexion que nous avons menée dans les parties précédentes. En effet, elle nous semble cristalliser les enjeux éthiques que nous avons évoqués et fournir un cadre conceptuel opératoire pour orienter le chercheur dans le traitement de données publiques recueillies sur Internet. Ainsi, l'étude de traces publiques de pratiques numériques nous semble requérir une attention particulière lorsque :

- le chercheur est susceptible d'augmenter le degré de publicité de ces inscriptions par sa recherche (que ce soit au moment de rendre compte de la recherche ou à l'étape de sa diffusion) ;

- le chercheur y a accès à la faveur de l'intervention d'un tiers qui a transformé les conditions particulières régissant leur publicité.

Le premier cas a été amplement illustré plus haut. II s'agit de l'effet « coup de projecteur » opéré par la recherche sur des écrits obscurs bien que publics. Le deuxième cas de figure mérite d'être davantage explicité. Un premier type de 


\section{Guillaume LATZKO-TOTH et Madeleine PASTINELLI}

situation, de plus en plus fréquent, survient quand des contenus archivés au su ou à l'insu des acteurs impliqués se trouvent publiés sous de nouvelles conditions d'accessibilité et de visibilité. Un exemple emblématique est la mise en ligne par Google d'un volume important de messages diffusés dans les forums Usenet. Or, à l'époque où ces écrits ont été publiés, l'archivage systématique des groupes Usenet n'existait pas. De plus, le contenu des groupes Usenet n'était pas indexé et ne pouvait donc faire l'objet d'une recherche «plein texte». Par conséquent, en compilant et en rendant disponibles ces contenus, Google en a profondément transformé la publicité, dans la mesure où cette dernière était essentiellement théorique et pour ainsi dire caduque jusqu'à ce qu'elle soit « réactivée ". II en va de même de certaines listes de discussion dont les messages s'adressant dans le contexte d'origine à une audience déterminée, se retrouvent un jour mis à disposition des chercheurs quand une archive de ces messages est publiée sur le web (voir Latzko-Toth et Proulx, 2013). Pour autant, il ne s'agit pas de messages privés puisque lesdites listes étaient pour la plupart publiques. II n'y a donc pas violation de la vie privée des acteurs, mais plutôt, en l'occurrence, une transformation de leur publicité.

Dans un cas comme dans l'autre, une amplification de la publicité des données est susceptible de découler des travaux de recherche. La question n'est pas de savoir si l'on doit s'abstenir de traiter ces données, mais plutôt de quelle manière le faire pour minimiser les conséquences négatives éventuelles de ces transformations pour les personnes impliquées. Puisque des écrits recueillis sur le web peuvent aisément être recontextualisés au moyen d'un moteur de recherche et que, de surcroît, la plupart des productions scientifiques contemporaines circulent sous forme numérique, on ne peut plus se contenter de masquer l'identité des acteurs en remplaçant leur nom ou leur identifiant par des pseudonymes ou des numéros. C'est le principe même de la citation d'extraits du corpus qui est en cause (Beaulieu et Estalella, 2012). Or, cela revient à remettre en question des façons de faire profondément enracinées dans les méthodes qualitatives, dont l'épistémologie de la preuve se fonde en grande partie sur la présentation au lecteur d'exemples suffisamment probants en qualité et en quantité pour que la thèse défendue soit jugée plausible par les pairs (Rueff, 2012). Ironiquement, les méthodes quantitatives de recherche en ligne reposant sur le traitement de masses de données (Big Data) semblent échapper à ces écueils, par le fait même qu'elles reviennent rarement au cas singulier et que les métadonnées générées dans le cours de l'analyse prennent le pas sur les données primaires ${ }^{20}$.

\footnotetext{
${ }^{20}$ Pour un aperçu de ces approches (digital methods et « tournant computationnel » des recherches en sciences sociales), voir Rogers (2009).
} 
Par-delà la dichotomie public/privé :

la mise en visibilité des pratiques numériques et ses enjeux éthiques

Plutôt que de renoncer au principe de l'exemple, certains méthodologues de la recherche qualitative en ligne préconisent le recours à l'exemple composite, produit en assemblant des fragments provenant de plusieurs cas (Barnes, 2004). Annette Markham a fait valoir que lorsque les données concernent des sujets sensibles ou des personnes vulnérables, l'invention d'exemples inspirés de cas réels est la seule approche permettant de garantir la sécurité des acteurs concernés (Markham, 2012). Et dans la mesure où les sciences humaines et sociales sont probablement appelées, à plus ou moins brève échéance, à faire le virage des données ouvertes à l'instar des autres sciences, il est concevable que les documents rendant compte de la recherche (rapports, articles) ne contiennent pas d'extraits du corpus, mais seulement des références précises à celui-ci, permettant aux pairs de contrôler par eux-mêmes le bien-fondé des assertions des chercheurs.

Cela dit, il importe aussi de prendre en compte la visibilité des véhicules de diffusion de la recherche et de mettre en balance les conséquences éventuelles de cette médiatisation relative avec les bienfaits pouvant résulter de la recherche. La diversité des situations et la singularité de chaque cas appellent une réflexion éthique ad hoc de la part des chercheurs, plutôt qu'une série de règles et de principes contraignants qui ne pourront jamais prendre en compte tous les cas de figure (voir Markham, 2006).

\section{Conclusion}

Par cet article, nous avons cherché à lever certaines ambiguïtés relatives à la catégorisation binaire des pratiques et traces numériques selon la dichotomie " public/privé ». II nous est en effet apparu que certains paradoxes et dilemmes éthiques auxquels sont confrontés les chercheurs proviennent de malentendus non seulement sur ces catégories, mais également sur les principes éthiques eux-mêmes. Ainsi, nous avons vu que la notion de consentement était sujette à des variations de sens, allant du consentement à participer à une étude au consentement à en être l'objet, mais que ces acceptions « maximalistes » du consentement des acteurs n'ont aucun rapport avec la nature privée ou publique des traces de leurs activités. Cela ne veut pas dire pour autant que la distinction public/privé soit caduque dans le cas d'interactions en ligne, ou que le respect de la vie privée des personnes cesse d'être un principe éthique fondamental. En revanche, nous espérons avoir établi que le «principe de précaution » voulant que l'on considère comme privées des inscriptions publiques du fait de leur caractère intime ou parce qu'elles ont été produites dans des contextes leur conférant une faible visibilité, n'est pas fondé. II s'agit plutôt d'être attentif à leur 


\section{Guillaume LATZKO-TOTH et Madeleine PASTINELLI}

façon d'être publiques, et de s'efforcer d'en préserver l'intégrité, ou du moins d'atténuer les conséquences de sa transformation pour les acteurs concernés. En particulier lorsque la publicité de ces pratiques est amplifiée par leur mise en visibilité. Enfin, il nous semble important de rappeler que ces altérations affectant la publicité des traces déposées dans les espaces numériques débordent le cadre des activités de recherche en sciences sociales.

\section{Références bibliographiques}

ABERCROMBIE N., LONGHURST B., 1998, Audiences: A Sociological Theory of Performance and Imagination, Londres, Sage.

BARNES S. B., 2004, "Issues of Attribution and Identification in Online Social Research », dans M. D. JOHNS, S. C. SHING-LING et G. J. HALL (dir.), Online Social Research : Methods, Issues, and Ethics, New York, Peter Lang, pp. 203-222.

BARNES S. B., 2006, «A privacy paradox: Social networking in the United States ", First Monday, vol. 11, n9, <http://firstmonday.org/article/view/1394/1312>, dernière consultation le 17 décembre 2013.

BAYM N. K., 1995, « The Emergence of Community in Computer-Mediated Communication ", dans S. G. JONES (dir.), CyberSociety. ComputerMediated Communication and Community, Thousand Oaks (Cal.), Sage Publications, pp. 138-163.

BAZIN J., 1998, "Questions de sens », L'Enquête, n6, pp. 13-34.

BAZIN J., 2008, Des clous dans la joconde. L'anthropologie autrement, Toulouse, Anacharsis.

BEAULIEU, A. et A. ESTALELLA, 2012, "Rethinking research ethics for mediated settings ", Information, Communication \& Society, vol. 15, $\mathrm{n}^{\circ} 1$, pp. 23-42.

BLAKE M., 2013, "The trouble with Torrington », Columbia Journalism Review, 25/03/2013, <http://www.cjr.org/

united_states_project/the_trouble_with_torrington.php $>$, dernière consultation le 27 mars 2013.

BOURDIEU P., 1980, Le sens pratique, Paris, Minuit.

BOYD D., 2006, « Facebook's 'Privacy Trainwreck': Exposure, Invasion, and Drama ", Apophenia Blog, publié le 08/09/2006, <http://www.danah.org/papers/FacebookAndPrivacy.html>, dernière consultation le 20 avril 2013. 
Par-delà la dichotomie public/privé :

la mise en visibilité des pratiques numériques et ses enjeux éthiques

BRIGHENTI A. M., 2010, «The Publicness of Public Space. On the Public Domain ", Quaderno, Vol. 49, Trento (Italie): Dipartimento di Sociologia e Ricerca Sociale, Università di Trento, <http://www.unitn.it/files/quad49.pdf>, dernière consultation le 29 mars 2013.

BRUCKMAN A., 2002, "Studying the amateur artist : A perspective on disguising data collected in human subjects research on the Internet », Ethics and Information Technology, vol. 4, $\mathrm{n}^{\circ}$ 3, pp. 217-231.

CARDON D., 2008, « Le design de la visibilité : un essai de cartographie du web 2.0 », Réseaux, n¹52, pp. 93-137.

CARDON D., 2012, « Le parler privé-public des réseaux sociaux d'Internet », dans S. PROULX, M. MILLETTE et L. HEATON (dir.), Médias sociaux : enjeux pour la communication, Québec, Presse de l'Université du Québec, pp. 33-45.

DAYAN D., 2000, «Télévision, le presque-public », Réseaux, n¹00, pp. 427456.

DAYAN D., 2002, « Les médias visuels et leurs publics ", Collège iconique du 05/02/2002, Bry-sur-Marne (France) : Institut national de l'audiovisuel, $<$ http://www.institut-national-audiovisuel.fr/sites/ina/medias/upload/actes-etparoles/colleges/2002/05-02-02_dayan.pdf>, dernière consultation le 26 janvier 2014.

EHRENBERG A., 1995, L'individu incertain, Paris, Calmann-Lévy.

EYSENBACH, G. et J. E. TILL, 2001, « Ethical issues in qualitative research on internet communities », BMJ, vol. 323, pp. 1103-1105, <http://www.bmj.com/content/323/7321/1103>, dernière consultation le 20 février 2014.

GIDDENS A., 1984, The constitution of society : outline of the theory of structuration, Berkeley, University of California Press.

GRADT J.-M., 2013, « La page Facebook « n'est pas un lieu public », juge la Cour de cassation », LesEchos.fr, 12 avril 2013.

JOLIE A., 2013, «My Medical Choice », The New York Times, 14/05/2013, p. A5, <http://www.nytimes.com/2013/05/14/ opinion/my-medicalchoice.html>, dernière consultation le 5 juillet 2013.

JOST F., 2002, L'empire du loft, Paris, La Dispute.

JOST F., 2009, Grandeur et misères de la télé-réalité, Paris, Le Cavalier bleu.

KAUFMANN J.-C., 2001, Ego. Pour une sociologie de l'individu, Paris, Nathan. 


\section{Guillaume LATZKO-TOTH et Madeleine PASTINELLI}

KING S. A., 1996, « Researching Internet Communities: Proposed Ethical Guidelines for the Reporting of Results ", The Information Society, vol. 12, n०2, pp. 119-128.

LAHIRE B., 1998, L'Homme pluriel. Les ressorts de l'action, Paris, Nathan.

LAHIRE B., 2013, Dans les plis singuliers du social ; individus, institutions, socialisations, Paris, La Découverte.

LAPLANTINE F., 1996, La description ethnographique, Paris, Nathan.

LATZKO-TOTH G. et S. PROULX, 2013, «Enjeux éthiques de la recherche sur le Web », dans C. BARATS (dir.), Manuel d'analyse du web en Sciences Humaines et Sociales, Paris, Armand Colin, pp. 32-48.

LAURENS S. et F. NEYRAT (dir.), 2010, Enquêter : de quel droit ? Menaces sur l'enquête en sciences sociales, Bellecombe-en-Bauges, Éditions du Croquant.

LEMIEUX C., 2009, Le devoir et la grâce, Paris, Économica.

LENCLUD G., 1995, «Quand voir, c'est reconnaître. Les récits de voyage et le regard anthropologique ", L'Enquête, vol. 1, n¹, pp. 113-129.

LIVINGSTONE, S., 2004, « Du rapport entre audiences et publics », Réseaux, $\mathrm{n}^{\circ} 126$, p. 17-55.

MARKHAM A. N., 2006, «Ethic as Method, Method as Ethic: A Case for Reflexivity in Qualitative ICT Research ", Journal of Information Ethics, vol. 15, n², pp. 37-54.

MARKHAM A. N., 2012, «Fabrication as Ethical Practice : Qualitative Inquiry in Ambiguous Internet Contexts ", Information, Communication \& Society, vol. 15, n`3, pp. 334-353.

MARTEL G., 2012, « La performance communicationnelle en contexte médiatique. L'exemple du débat politique télévisé ", Mots. Les langages $d u$ politique, $\mathrm{n}^{\circ} 92$, <http://mots.revues.org/19532>, dernière consultation le 29 novembre 2013.

MARTUCCELLI D., 2002, Grammaires de l'individu, Paris, Gallimard.

McKEE H. A. et J. E. PORTER, 2009, The Ethics of Internet Research. A Rhetorical, Case-Based Process, New York, Peter Lang.

MEHL D., 1996, La télévision de l'intimité, Paris, Seuil.

MELTZ R., 2009, « Marc L. Genèse d’un buzz médiatique », Le Tigre, n`30, pp. 14-16, <http://www.le-tigre.net/Marc-L-Genese-d-un-buzzmediatique.html>, dernière consultation le 5 juillet 2013. 
Par-delà la dichotomie public/privé :

la mise en visibilité des pratiques numériques et ses enjeux éthiques

MILLERAND F., 2003, L'appropriation du courrier électronique en tant que technologie cognitive chez les enseignants chercheurs universitaires. Vers l'émergence d'une culture numérique ?, thèse de doctorat en communication, Université de Montréal, $<h t t p: / / w w w . t h e s e s . u m o n t r e a l . c a / t h e s e s /$ nouv/millerand_f/these.pdf>, dernière consultation le 15 juin 2013.

MONTJOYE (de) Y.-A., C. A. HIDALGO, M. VERLEYSEN et V. D. BLONDEL, 2013, "Unique in the Crowd: The privacy bounds of human mobility », Scientific Reports, vol. 3, article n 1376 , <http://www.nature.com/srep/2013/130325/srep01376/ full/srep01376.html>, dernière consultation le 5 juillet 2013.

NISSENBAUM H., 2004, «Privacy as Contextual Integrity », Washington Law Review, vol. 79, n¹, pp. 119-157.

PASTINELLI M., 2007, Des souris, des hommes et des femmes au village global, Québec, Presses de l'Université Laval.

PISANI F., 2012, «Enswers : le Google de la vidéo qui vient de Corée », Winch5 (Les blogs du Monde), 20/09/2012, <http://winch5.blog.lemonde.fr/2012/09/20/enswers-le-google-de-la-videoqui-vient-de-coree/>, dernière consultation le 29 novembre 2013.

RALLET A. et F. ROCHELANDET, 2011, «Présentation », Réseaux, n¹67, pp. 9-16.

RICOEUR P., 1988, Philosophie de la volonté. Finitude et culpabilité, Paris, Aubier.

ROBERTS L., L. SMITH et C. POLLOCK, 2004, « Conducting Ethical Research Online: Respect for Individuals, Identities and the Ownership of Words ", dans E. A. BUCHANAN (dir.), Readings in Virtual Research Ethics : Issues and Controversies, Hershey, Penn., Information Science Publishing, pp. 156173.

ROGERS R., 2009, The End of the Virtual: Digital Methods, Amsterdam, Amsterdam University Press.

ROUQUETTE S., 2008, « Les blogs « extimes » : analyse sociologique de l'interactivité des blogs », tic\&société, vol. 2, n¹, <http://ticetsociete.revues.org/412>, dernière consultation le 15 juin 2013.

RUEFF J., 2012, «Quelques éléments d'épistémologie concernant les recherches qualitatives et critiques en communication ", Revue internationale de communication sociale et publique, $\mathrm{n}^{\circ} 7$, pp. 23-40. 


\section{Guillaume LATZKO-TOTH et Madeleine PASTINELLI}

SEC, 2013, « SEC Says Social Media OK for Company Announcements if Investors Are Alerted », communiqué de presse 2013-51, http://www.sec.gov/news/press/2013/2013-51.htm>, dernière consultation le 5 juillet 2013.

SMITH A., 2014, « 6 new facts about Facebook », Pew Research Center, publié le 03/02/2014, <http://www.pewresearch.org/ fact-tank/2014/02/03/6-newfacts-about-facebook/>, dernière consultation le 26 janvier 2014.

SVENINGSSON M., 2008, " How Do Various Notions of Privacy Influence Decisions in Qualitative Internet Research? ", dans A. N. MARKHAM et N. K. BAYM (dir.), Internet Inquiry: Conversations About Method, London, Sage Publications, pp. 69-87.

THOMPSON J. B, 2000, «Les transformations de la visibilité », Réseaux, n¹00, pp. 189-213.

TISSERON S., 2011, "Intimité et extimité », Communications, n88, pp. 83-91.

TRUDEL P. et M. JEAN (dir.), 2010, La Malréglementation. Une éthique de la recherche est-elle possible et à quelles conditions ?, Montréal, Presses de I'Université de Montréal.

VINCENT D., O. TURBIDE et M. LAFOREST, 2008, La radio X, les médias et les citoyens : dénigrement et confrontation sociale, Québec, Nota Bene.

WALTHER J., 1996, « Computer-Mediated Communication: Impersonal, Interpersonal and Hyperpersonal Interaction », Communication Research, vol. 23, n¹, pp. 3-43.

ZIMMER, M., 2010, “'But the data is already public': on the ethics of research in Facebook », Ethics and Information Technology, vol. 12, n² 4, pp. 313-325. 